\title{
Implementation of a New and Modified Scheme of Butler Matrix for C-Band Applications with Enhanced Characteristics
}

\author{
Mostafa MALEKI, Javad NOURINIA, Changiz GHOBADI, Rahim NADERALI \\ Dept. of Electrical Engineering, Urmia University, Urmia, Iran \\ \{m.maleki, j.nourinia, ch.ghobadi,r.naderali\}@urmia.ac.ir
}

Submitted December 28, 2019 / Accepted March 24, 2020

\begin{abstract}
In this paper, the structure of a miniaturized broadband $4 \times 4$ Butler matrix is presented. All components of the proposed feeding network are designed in $a$ way that they have the smallest electrical size and acceptable performance in the $C$ band. Despite conventional Butler matrix consists of phase shifters, the proposed network benefits from dummy crossover, which leads to improving the phase difference bandwidth. Since in beam switching networks, $90^{\circ}$ coupler has a vital role, the main focus is concentrated on the aforementioned component as mentioned above. The compactness of the proposed coupler is associated with embedding the S-shaped arms instead of ordinary elements. Due to overcoming the problem of a mismatch phase difference between phase shifter and crossover, the modified dummy crossover is used. In order to improve the overall performance of the depicted feeding network, S-shaped electromagnetic bandgap structures are used between elements. They reduce destructive mutual coupling effect hence leads to enhance total network efficiency. The extracted results determine that the bandwidth of the presented network is from 3.5 8.2 GHz that covers the $C$ band totally.
\end{abstract}

\section{Keywords}

Butler matrix, coupler, broadband, electromagnetic band gap

\section{Introduction}

Butler matrices are popular multi-input multi-output circuits in microwave components. They are beam switching networks capable of producing orthogonal beams, which are essential for designing complex and smart radiating systems. $N \times N$ Butler matrix ( $N$ input ports and $N$ output ports) is a passive microwave network. If an $N \times N$ Butler matrix is used to feed an N-element array antenna, a set of $N$ orthogonal beams will be generated [1]. These matrices generally are composed of hybrids, crossovers, and phase shifters. Due to the increment of subscribers, demand to the enhancement of transmission capacity, and reduction of multipath fading, the design should be optimal for our purpose. Therefore, the performance of individual network elements should be improved as much as possible. Generally, there are two approaches to achieve a broadband Butler matrix. The first method is using multilayer structures such as coplanar waveguide (CPW) [2] and substrate integrated waveguide (SIW) technology [3], [4]. The second approach is based on microstrip and stripline circuits [5]. In the study of the second method, some papers were published based on modified hybrids and synthetic branch-line couplers. In the case of improving the total bandwidth of the feeding network, the elements should be designed as broadly as possible. On the other hand, miniaturization should not be neglected. In the case of couplers that are basic elements of Butler matrices, attached open stubs lead to having a compact size and stable relativity impedance matching with frequency variations. The Butler matrix introduced in [6] is made of modified $3 \mathrm{~dB}$ branch-line couplers for $5 \mathrm{G}$ applications, which have broadband performance. In [7], [8] $3 \mathrm{~dB}$ branch-line couplers with additional stubs were shown to achieve the minimal electrical size and acceptable wideband performance simultaneously. Due to attached arms modeled as distributed capacitance, they are capable of declining physical size of coupler while the electrical length is maintained. On the other hand, the interaction of mentioned arms (role as capacitors) between different current paths (role as inductors) results to generate new resonate frequency and accordingly leads to improvement of impedance matching. However, for crossover investigation, conventional models suffer from narrow bandwidth and electrical size [9]. In order to overcome the mentioned first inherent limitation, using cascade topology seems to be effective. The minimization process is exactly similar to hybrid compression. The existence of unwanted surface currents, especially in monolithic microwave integrated circuits (MMIC), results in destructive mutual coupling phenomena that dramatically reduce overall efficiency. The utilization of electromagnetic bandgap (EBG) structure is an effective approach to overcome the problems of surface and leaky waves [10]. Different types of EBG schemes have 


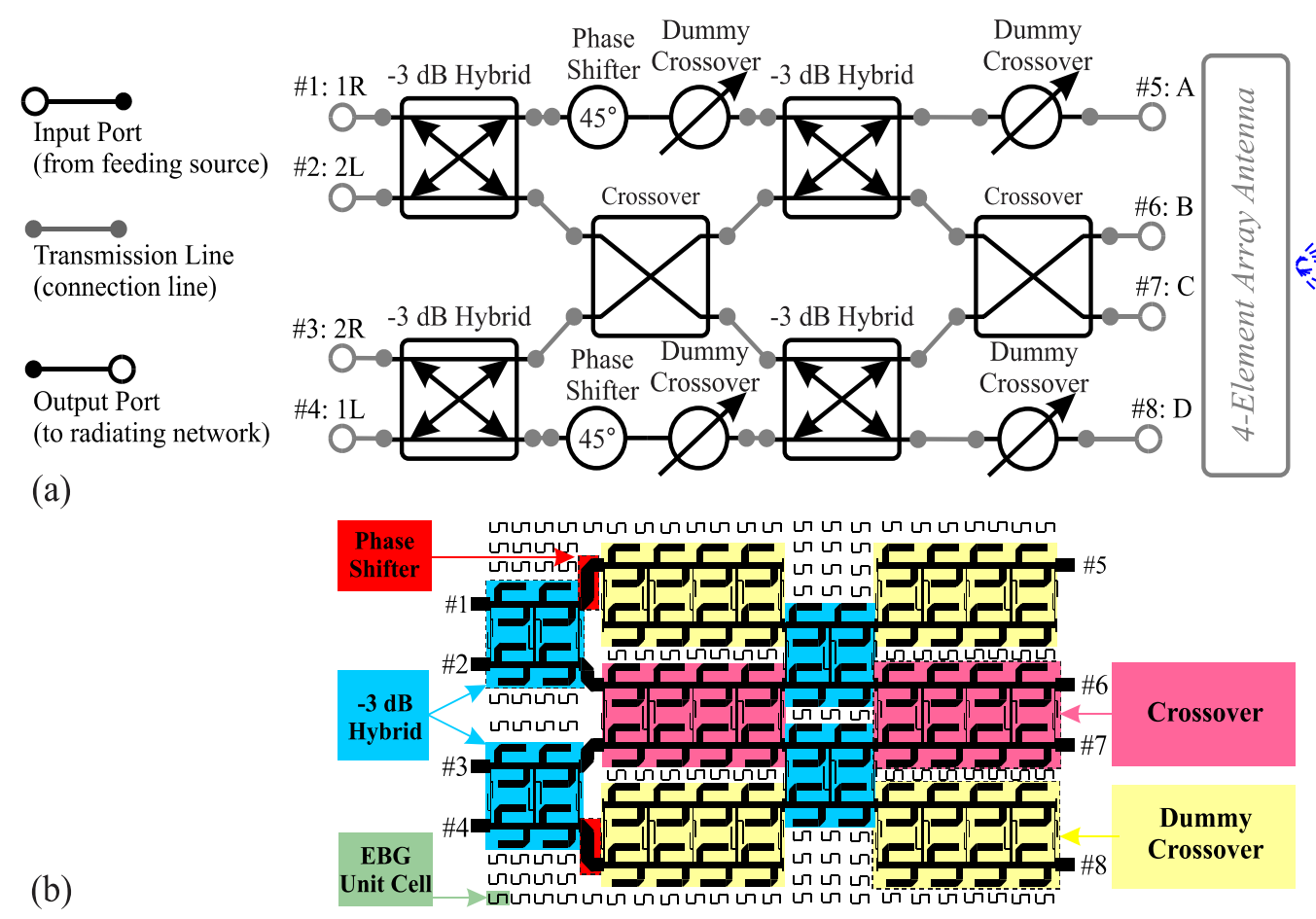

Fig. 1. (a) Block diagram, (b) schematic of the proposed Butler matrix.

\begin{tabular}{|c|c|c|c|c|c|}
\hline $\begin{array}{c}\text { Output } \\
\text { Input }\end{array}$ & \#5: A & \#6: B & \#7: C & \#8: D & $\boldsymbol{\Delta \varphi}$ \\
\hline$\# 1: 1 \mathrm{R}$ & $-45^{\circ}$ & $-90^{\circ}$ & $-135^{\circ}$ & $-180^{\circ}$ & $-45^{\circ}$ \\
\hline$\# 2: 2 \mathrm{~L}$ & $-135^{\circ}$ & $0^{\circ}$ & $-225^{\circ}$ & $-90^{\circ}$ & $135^{\circ}$ \\
\hline$\# 3: 2 \mathrm{R}$ & $-90^{\circ}$ & $-225^{\circ}$ & $0^{\circ}$ & $-135^{\circ}$ & $-135^{\circ}$ \\
\hline$\# 4: 1 \mathrm{~L}$ & $-180^{\circ}$ & $-135^{\circ}$ & $-90^{\circ}$ & $-45^{\circ}$ & $45^{\circ}$ \\
\hline
\end{tabular}

Tab 1. Phase distribution of output ports according to input ports.

been considered in [11]. It is evident, when a planar microstrip microwave layout is fabricated on an EBG substrate, the impedance bandwidth is enhanced. In this work, $\mathrm{S}$-shaped metamaterial array is designed in a way that they have band stop at $5.5 \mathrm{GHz}$ (WLAN) and produce a current virtual dam.

\section{Design Process}

Figure 1(a) illustrates the block diagram of a $4 \times 4$ Butler matrix proposed in this paper. Table 1 depicts the phase distribution of output ports for stimulates of input ports. Figure 1(b) shows the modified layout of broadband and miniaturized feeding network based on the Butler matrix design. It is obvious it consists of two pairs of broadband hybrid couplers and dummy crossovers, two $45^{\circ}$ phase shifters, a pair of crossovers, and an S-shaped array of electromagnetic bandgap structures. The design of broadband components results from having relatively wideband performance. (a)

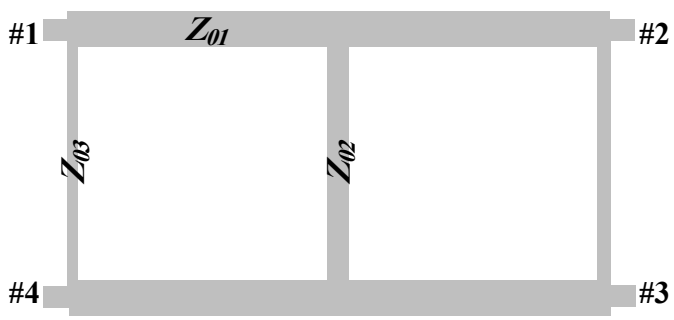

(b)

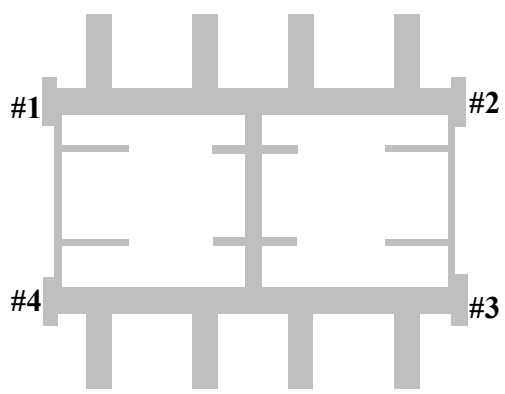

\#3

(c)

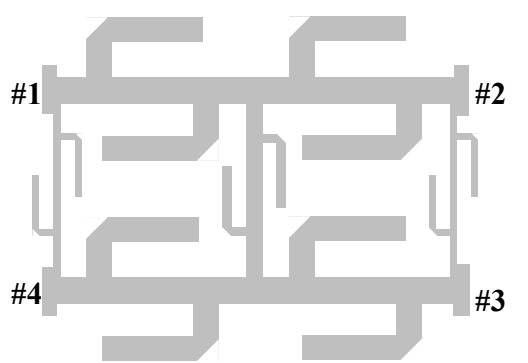

Fig. 2. Design process: (a) Double-box, (b) quasi $\pi$-shaped and (c) quasi S-shaped coupler. 


\subsection{3-Branch-line $3 \mathrm{~dB}$ Couplers}

Due to achieving a miniaturized and broadband structure, S-shaped 3-branch-line couplers are substituted by ordinary $90^{\circ}$ hybrid [12]. The output results of optimized $3 \mathrm{~dB}$ couplers such as reflection coefficient and isolation should be better than $15 \mathrm{~dB}$ over $80 \%$ or wider bandwidth. The design process of the proposed hybrid coupler was investigated in three iterations as shown in Fig. 2.

Step 1: Coupler 1; To enhance the bandwidth, cascade configuration is used as a double box. Figure 3 shows the extracted results of this coupler. As shown, the impedance bandwidth is between $4.2 \sim 6.7 \mathrm{GHz}(45 \%)$, and the linear phase difference is obtained at the mentioned band. The physical size of coupler is $12.3 \times 16.8 \mathrm{~mm}^{2}$.

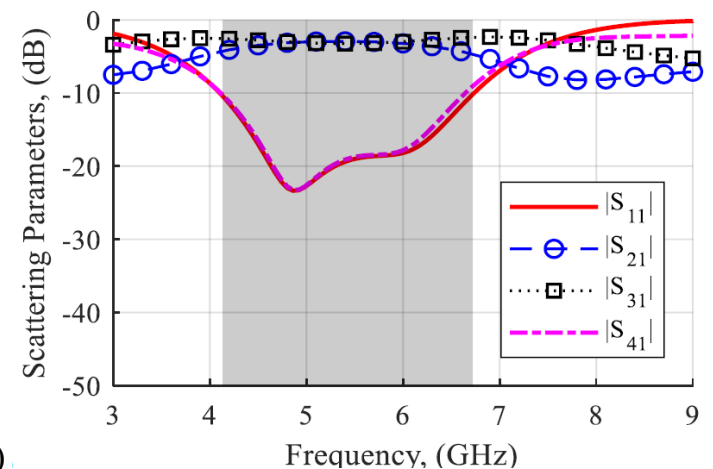

(a)

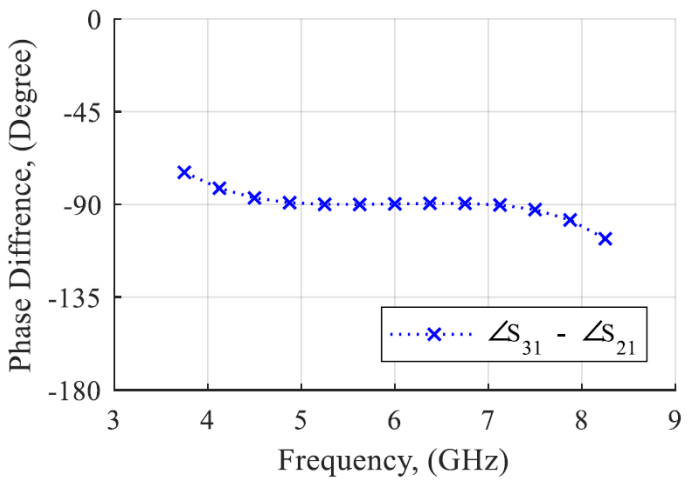

Fig. 3. (a) Scattering parameters, (b) phase difference of the double box $90^{\circ}$ coupler.

Step 2: Coupler 2; For better tuning of impedance matching and minimizing the physical size, vertical and horizontal arms as open circuit (O.C.) stubs are added to the previous step. A transmission line $\left(Z_{0}, \theta_{0}\right)$ and its $\pi$ equivalent circuit are shown in Fig. 4(a), and the design equations can be defined as follows [1]:

$$
\begin{gathered}
\frac{\tan \theta_{\mathrm{P}}}{Z_{\mathrm{P}}}=\frac{\cos \theta_{\mathrm{S}}-\cos \theta_{0}}{Z_{0} \sin \theta_{\mathrm{S}}}, \\
Z_{\mathrm{S}}=\frac{Z_{0} \sin \theta_{0}}{\sin \theta_{\mathrm{S}}}
\end{gathered}
$$

where $0 \leq \theta_{\mathrm{S}} \leq \theta_{0} \leq 90^{\circ}$. (a)

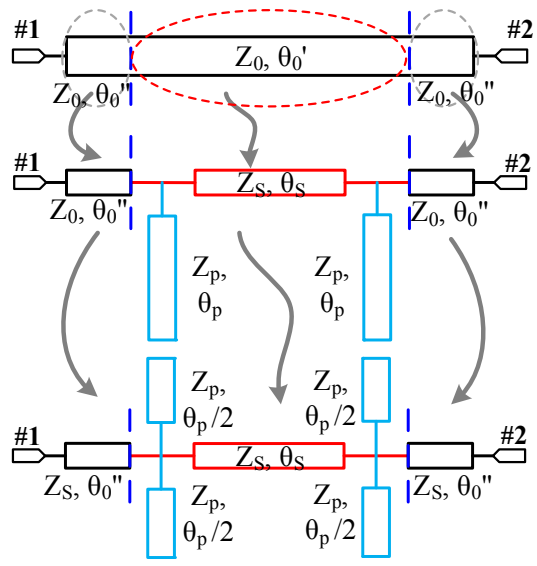

(b)
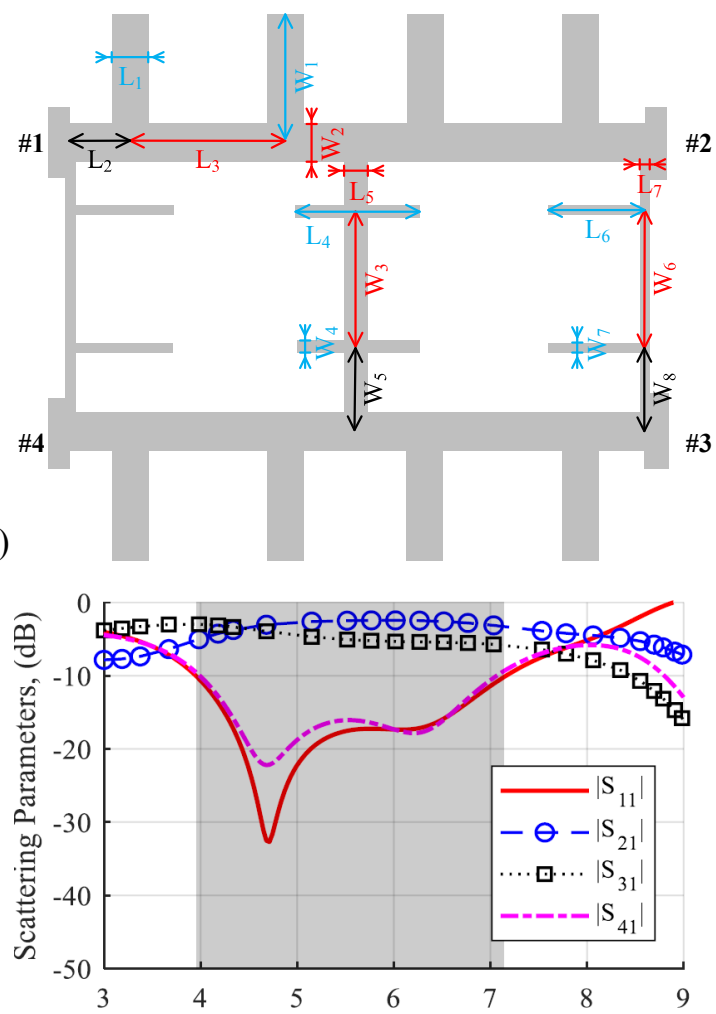

(c)

Frequency, $(\mathrm{GHz})$

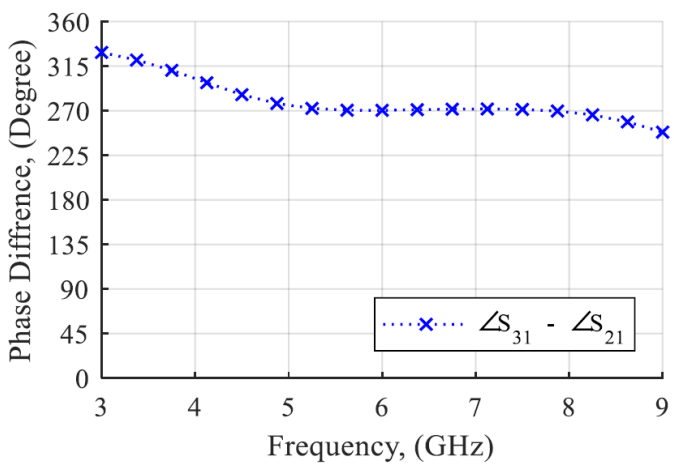

Fig. 4. (a) Scheme of the proposed quasi $\pi$-shaped equivalent of transition line, (b) layout, (c) scattering parameters, (d) phase difference. The quasi $\pi$-shaped coupler dimensions in (b) are (units: $\mathrm{mm}$ ): $L_{1}=8, L_{2}=0.8$, $L_{3}=3.4, L_{4}=3, L_{5}=0.3, L_{6}=2.4, L_{7}=0.1, W_{1}=0.8, W_{2}=4$, $W_{3}=4, W_{4}=0.2, W_{5}=1.4, W_{6}=4, W_{7}=0.1, W_{8}=1.4$. 


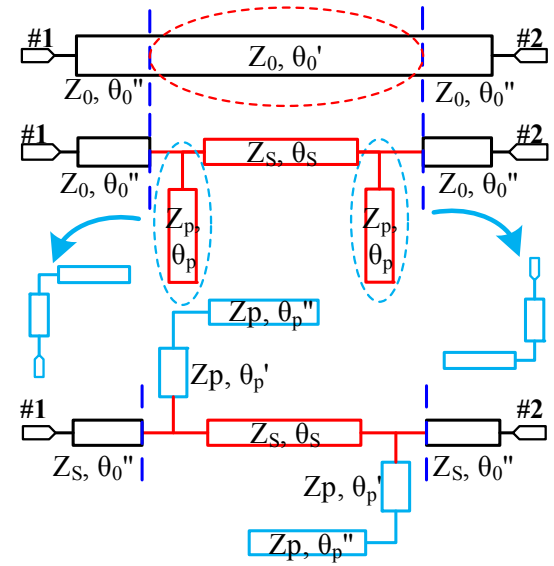

(a)
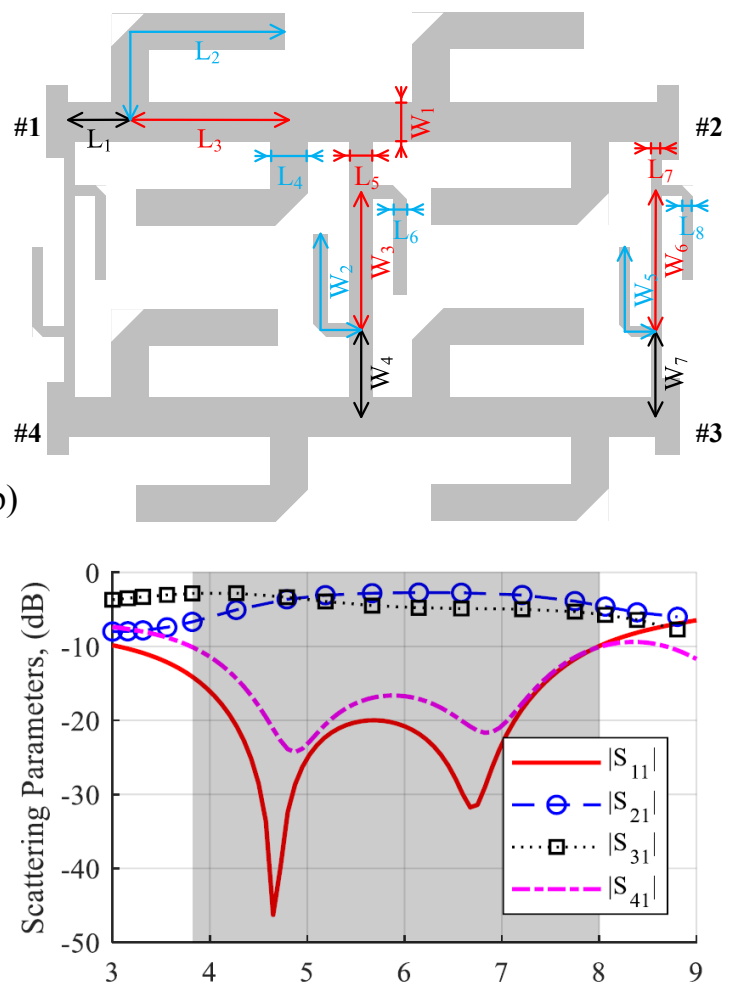

(c)

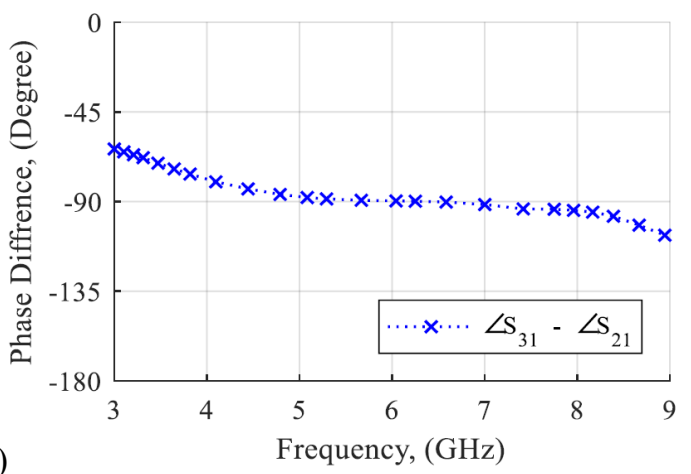

Fig. 5. (a) Scheme of the quasi S-shaped equivalent of transition line, (b) layout, (c) scattering parameters, (d) phase difference of the proposed quasi S-shaped $90^{\circ}$ coupler. The quasi S-shaped coupler dimensions in (b) are (units: mm): $L_{1}=0.8, L_{2}=5, L_{3}=3.4, L_{4}=0.7$, $L_{5}=0.3, L_{6}=0.2, L_{7}=0.1, L_{8}=0.1, W_{1}=0.8, W_{2}=3, W_{4}=1.4$, $W_{5}=2, W_{5}=4, W_{7}=1.4, W_{8}=4$
It's mentioned that, in comparison of the previous step, the overall size is reduced by approximately $40 \%$ $\left(11.5 \times 10.7 \mathrm{~mm}^{2}\right)$. Figure 4 shows the equivalent circuit and simulated scattering parameters. The impedance bandwidth is achieved between $3.9 \sim 7.2 \mathrm{GHz}(60 \%)$. It's clear that despite remarkable size reduction, impedance bandwidth is improved approximately $15 \%$.

Step 3: Coupler 3; Due to resolve the destructive interaction between attached stubs at array from among adjacent components, these additional arms are modified as a quasi S-shaped structure. Since these stubs result in producing additional lumped capacitance hence new resonant frequency is generated (at $6.8 \mathrm{GHz}$ ), and impedance bandwidth is enhanced. Figure 5 illustrates the property and results of this coupler. Impedance bandwidth is between $3.9 \sim 7.9 \mathrm{GHz}(69 \%)$ and supports $\mathrm{C}$ operational band approximately. This modified coupler has $41 \%$ size reduction with respect to conventional coupler and its dimensions are $11.5 \times 10.5 \mathrm{~mm}^{2}$.

\subsection{Crossovers}

At the realization of a planar Butler matrix, crossovers are necessary components. Figure 6 shows the geometry of optimized crossover which is obtained by cascading two couplers. As shown in Fig. 6(a), two S-shaped hybrids are attached consecutively. The optimal operation of the desired crossover can be achieved by adjusting the size of the interconnections. The S-parameters after optimization using the software Advanced Design system (ADS) are shown in Fig. 6(b).
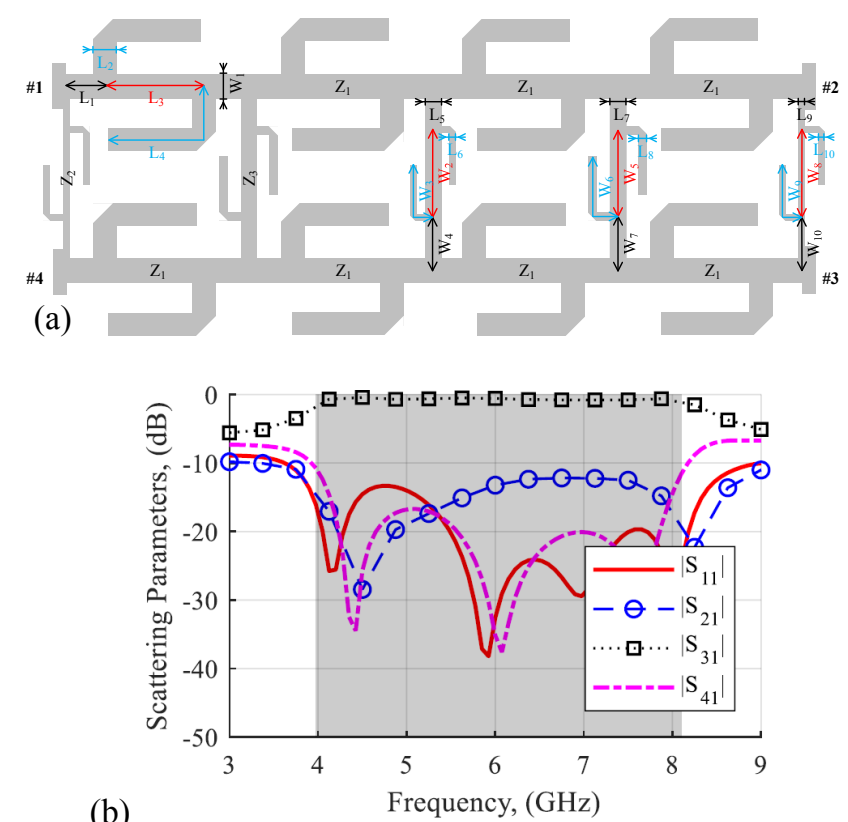

Fig. 6. (a) The layout, (b) scattering parameters. The quasi Sshaped crossover dimensions in (a) are (units: $\mathrm{mm}$ ): $L_{1}=0.8, L_{2}=0.8, L_{3}=3.4, L_{4}=5, L_{5}=0.1, L_{6}=0.1, L_{7}=0.3$, $L_{8}=0.2, L_{9}=0.1, L_{10}=0.1, W_{1}=0.7, W_{2}=4, W_{3}=2, W_{4}=1.4$, $W_{5}=4, W_{6}=3, W_{7}=1.4, W_{8}=4, W_{9}=2, W_{10}=1.4$. 


\subsection{Phase Shifters}

Implementation of the differential phase shifter by a traditional transmission line leads to a limited operational band. Using the principle of Schiffman phase shifter, the broadband structure could be achieved. Also, the desired phase difference can be realized using dummy crossovers as phase shifters. The existence of ordinary crossovers results in generating unwanted phase difference (which is not completely linear as shown in Fig. 7(c)), and embedding dummy crossover at the final scheme leads to compensation of this phenomena (without inserting any transmission line with micro and macro bends which can lead to a impedance mismatch).

The schematic and extracted results of dummy crossover are shown in Fig. 7. The required phase difference for the modified feeding network is reached, as illustrated clearly in Fig. 7.
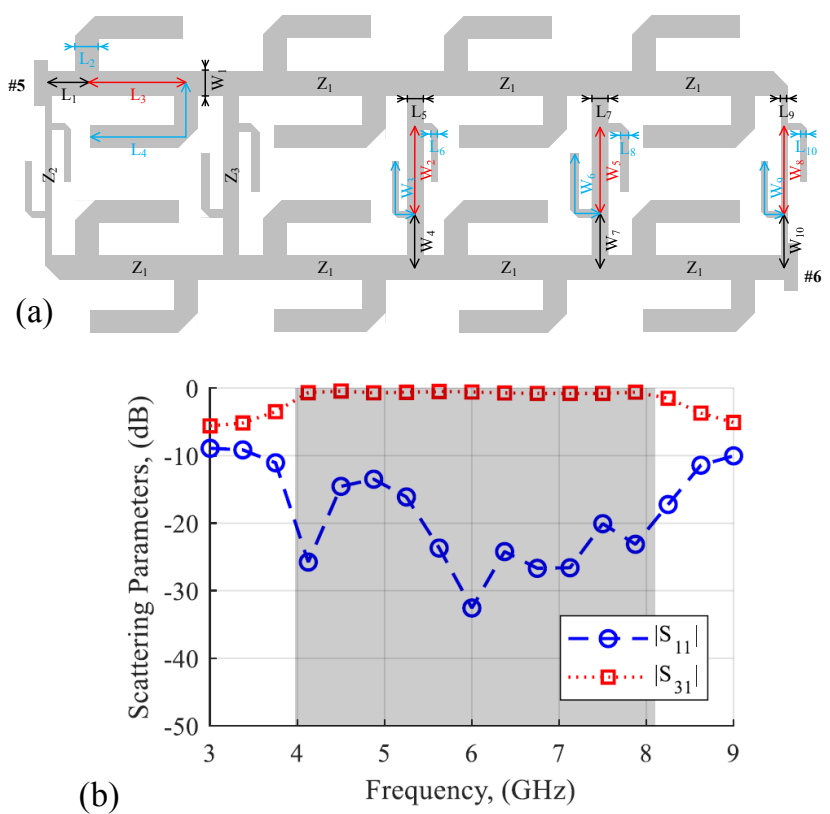

(b)

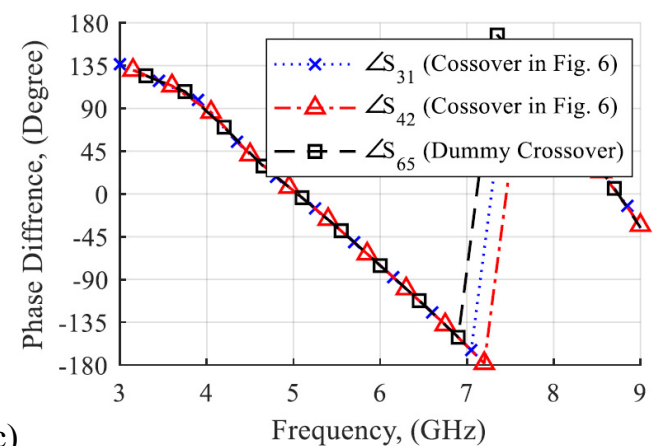

Fig. 7. (a) The layout, (b) scattering parameters of the proposed dummy crossover and (c) phase difference compression of the crossover and dummy crossover. The quasi S-shaped crossover dimensions in (a) are (units: $\mathrm{mm}$ ): $L_{1}=0.8, L_{2}=0.8, L_{3}=3.4, L_{4}=5, L_{5}=0.1$, $L_{6}=0.1, L_{7}=0.3, L_{8}=0.2, L_{9}=0.1, L_{10}=0.1, W_{1}=0.7, W_{2}=4$, $W_{3}=2, W_{4}=1.4, W_{5}=4, W_{6}=3, W_{7}=1.4, W_{8}=4, W_{9}=2$, $W_{10}=1.4$.

\subsection{Electromagnetic Bandgap (EBG) Structure}

The existence of the unwanted surface waves among the microwave component results in intense mutual coupling, which reduces the array operation. Recently, different kinds of EBG structures have been studied in microwave components. Mushroom-like model is one of the popular types, and it is fabricated by small metal pads with the grounding vias. Uni-planar compact EBG (UCEBG) structures are made of square metallic patches connected by strips mounted on grounded dielectric slab to form an LC network. Traditional UCEBG scheme is composed of square pads and narrow lines with insets. The gap between the two near unit cells acts as capacitance, and the strips with insets illustrate the inductance. For better miniaturization, the equivalent capacitance and inductance must be increased. To verify the properties of EBG structures and determining the band-gap regions, a $2 \times 4$ array of $\mathrm{S}$ shaped EBG structures is inserted between the two $50 \Omega$ open-ended microstrip-line as shown in Fig. 8(a). The EBG structures are fabricated on Rogers RO4003 substrate with permittivity of 3.55 while thickness is $0.8 \mathrm{~mm}$. The simulated result for the transmission coefficient is demonstrated in Fig. 8(b). The existence of EBG schemes among microstrip lines results in to significant reduction of $S_{21}$ magnitude at two ranges of $4.85 \sim 6.1 \mathrm{GHz}$ and $6.8 \sim 7.4 \mathrm{GHz}$. It is clear that the mutual coupling between feeding network elements is effectively reduced with EBG structure.

(a)

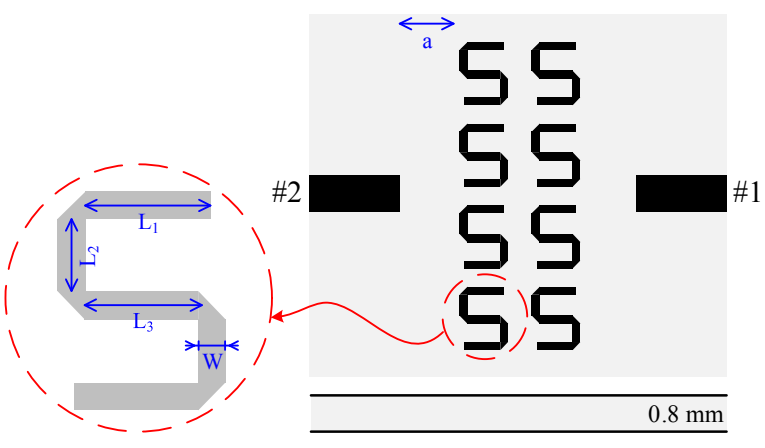

(b)

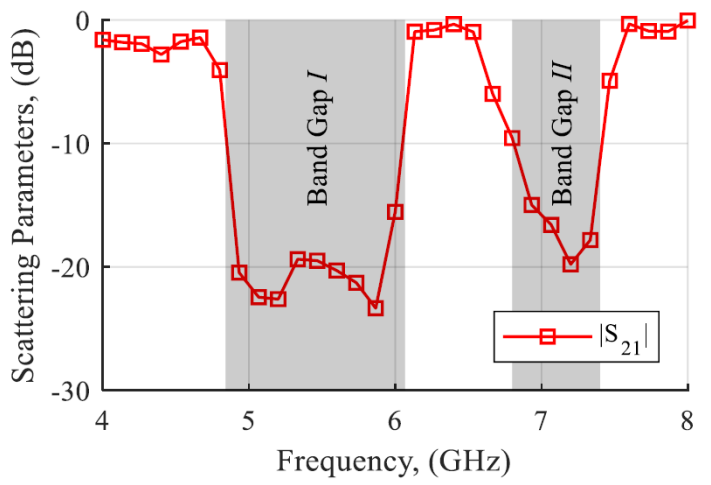

Fig. 8. (a) The layout, (b) scattering parameters of the proposed EBG. The EBG dimensions in (a) are (units: $\mathrm{mm}): L_{1}=0.9, L_{2}=0.8, L_{3}=0.8, W=0.2, a=1$. 


\section{Results and Discussions}

The presented modified Butler matrix was realized and measured in order to validate the design process. Figure 9 demonstrates the photograph of the fabricated feeding network. Its dimension is $43 \times 71 \times 0.8 \mathrm{~mm}^{3}$ and is

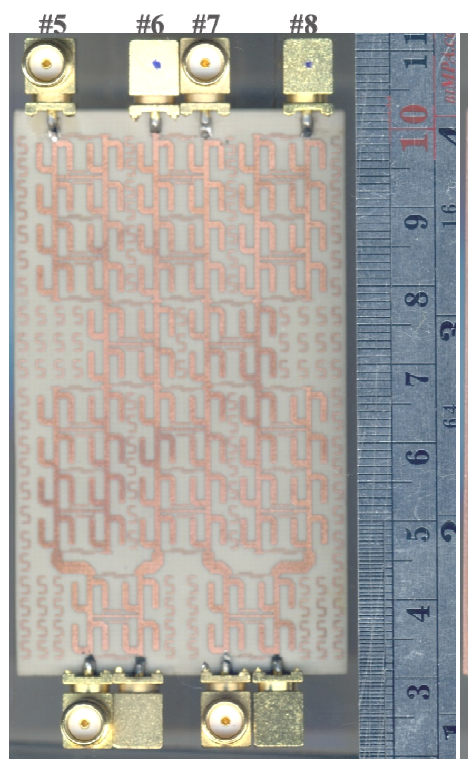

(a)

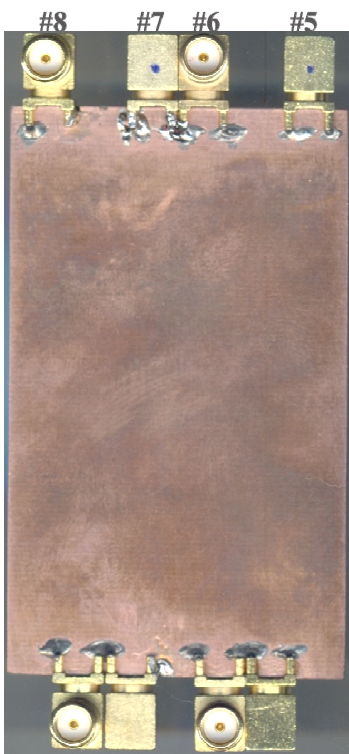

(b)
\#4 \#3 \#2 \#1
Fig. 9. The photograph of the proposed $4 \times 4$ Butler matrix (a) top layer, (b) bottom layer.
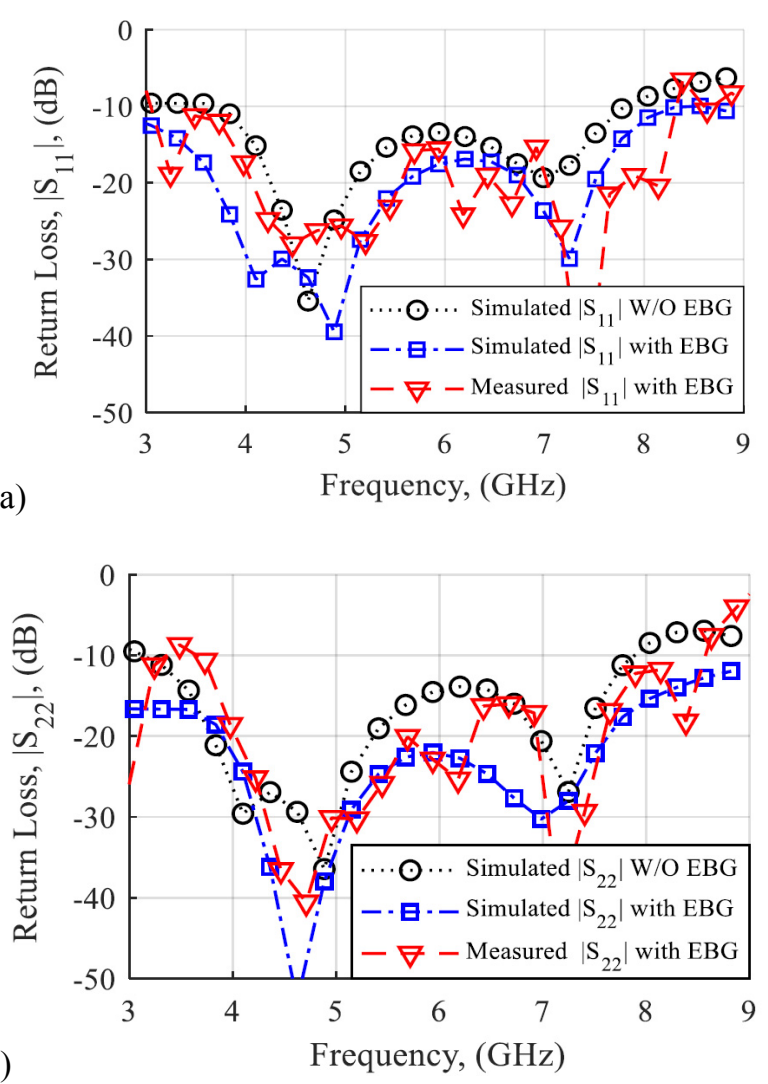

Fig. 10. Simulated and measured results of return-loss for feeding network when (a) port 1 and (b) port 2 is fed.
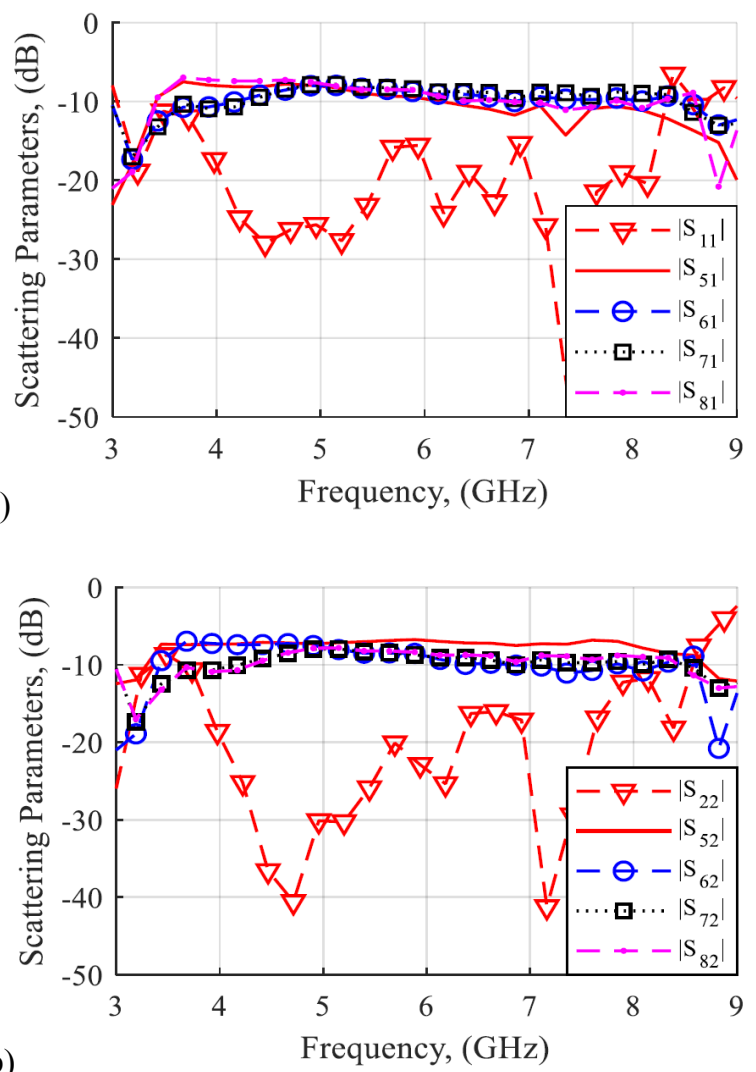

(b)

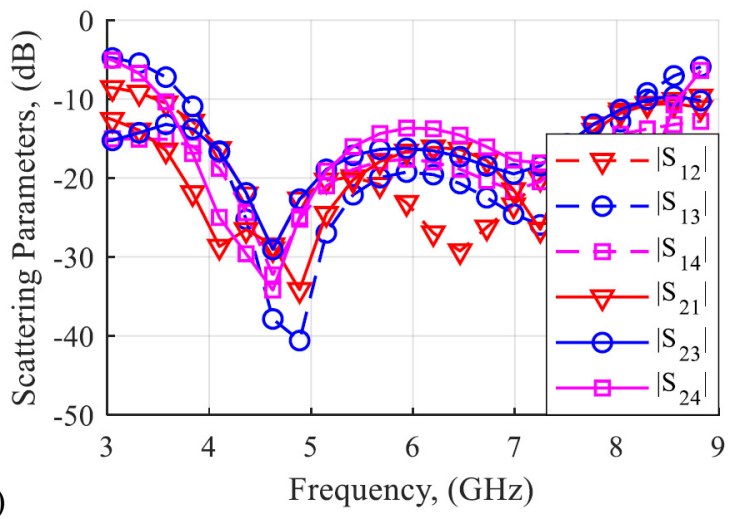

Fig. 11. Scattering parameters, (a) port 1, (b) port 2 and (c) isolation of the input ports.

implemented on Rogers RO4003. Scattering parameters were measured by the Agilent ${ }^{\mathrm{TM}} 8363 \mathrm{C}$ network analyzer. Since the network has approximately symmetrical structures, only two ports (1 and 2) were measured in the laboratory. Extracted results of scattering parameters for mentioned ports for two states (state 1: with and state 2: without (EBG)) are illustrated in Fig. 10(a) and (b), respectively. It's clear that when port 1 is excited the impedance bandwidth covers the frequency range of $4 \sim 7.5 \mathrm{GHz}$ (state 1) and $3.5 \sim 8 \mathrm{GHz}$ for state 2. These values are between $3.9 \sim 7.7 \mathrm{GHz}$ (state 1) and $3.5 \sim 8.2 \mathrm{GHz}$ (state 2) for port 2 stimulus. Electromagnetic bandgap structures lead to approximately $18 \%$ bandwidth enhancement. The transmission coefficient curves have acceptable adaptability with reflection coefficient values for both 
exciting ports, see Fig. 11. The differential phase of output ports for the port $1 \& 2$ was shown in Fig. 12(a). It's noticed that when port 1 is excited the phase difference among output ports is approximately equal to $-45^{\circ}$. It's also obvious that for port 2 stimulus, the differential phase is $\approx 135^{\circ}$. Based on extracted results for two ports excitation and design process, it seems that the proposed modified network is a good candidate for broadband standard Butler matrix application. To confirm this claim, we need to output results for the port $3 \& 4$. It is necessary to mention only simulated results were discussed for these ports. Figure 12(b) depicted that the differential phase of output ports are $-135^{\circ}$ and $45^{\circ}$ for port $3 \& 4$, respectively.

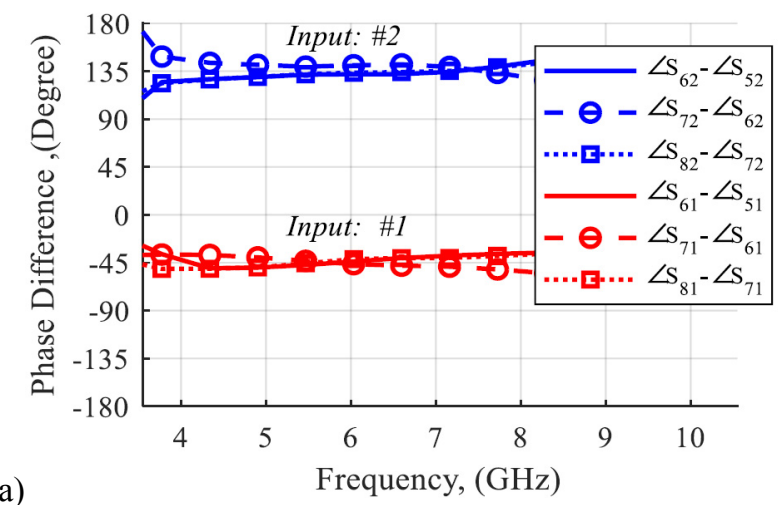

(a)

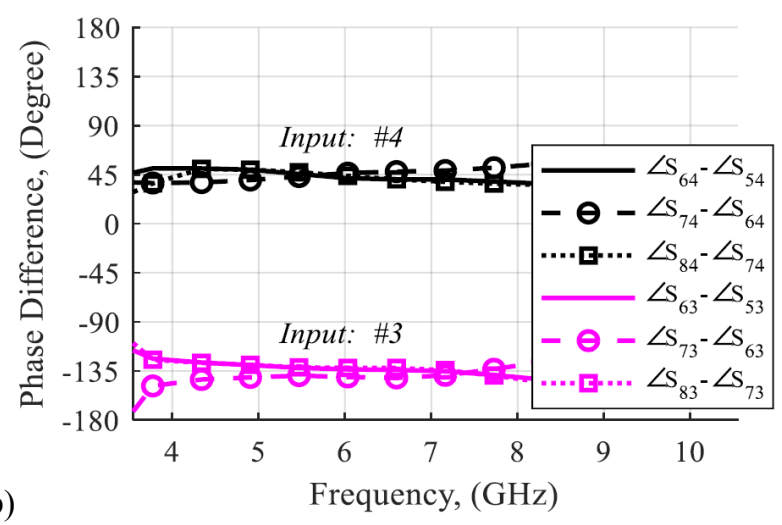

Fig. 12. Phase differences for feeding network when (a) port $1 \& 2$ and (b) port $3 \& 4$ are fed.

\begin{tabular}{|c|c|c|}
\hline Proposed Structures & Operational Band & Physical Size $\left(\mathbf{m m}^{\mathbf{2}}\right)$ \\
\hline$[13]$ & $5 \sim 8 \mathrm{GHz}$ & $121 \times 194$ \\
\hline$[10]$ & $4.1 \sim 7 \mathrm{GHz}$ & $90 \times 127$ \\
\hline$[14]$ & $4 \sim 7.9 \mathrm{GHz}$ & $110 \times 110$ \\
\hline Presented Network & $3.5 \sim 8.2 \mathrm{GHz}$ & $43 \times 71$ \\
\hline
\end{tabular}

Tab 2. The comparison table between presented network and previously published structures.

The comparison table between presented network and previously published structures is introduced; it seems that the presented network covers $\mathrm{C}$ - band totally and a portion of standard WMAX $(3.5 \mathrm{GHz})$; while its physical size is significantly compact with respect to other works.

\section{Conclusion}

A compact modified Butler matrix was proposed, realized, and measured to validate its design process. The structure of the presented network has a uniform scheme composed of S-shaped modified hybrid couplers, crossovers, phase shifters, and electromagnetic bandgap structures. At the first step, due to the optimization of the network, changes have been made at components structures. To have acceptable transmission operation, dummy crossover is designed as phase shifters, which implies accurate phase shifts as crossovers. At the second step, the array of electromagnetic bandgap structures is embedded among network components to decrease surface wave malicious effect. The feeding network has impedance bandwidth of over a frequency range of $3.5 \sim 8.2 \mathrm{GHz}$ that covers $\mathrm{C}$ band totally. The proposed Butler matrix could advantageously be used as a beam-forming network for a wideband antenna array.

\section{References}

[1] CHEN, C., WU, H., WU, W. Design and implementation of a compact planar 4x4 microstrip Butler matrix for wideband application. Progress in Electromagnetics Research C, 2011, vol. 24, p. 43-55. DOI: 10.2528/PIERC11072614

[2] NEDIL, M., DENIDNI, T. A., TALBI, L. Novel Butler matrix using CPW multilayer technology. IEEE Transactions on Microwave Theory and Techniques, 2006, vol. 54, no. 1, p. 499-507. DOI: 10.1109/TMTT.2005.860490

[3] ZHENG, S., CHAN, W. S., LEUANG, S. H., et al. Broadband Butler matrix with flat coupling. Electronics Letters, 2007, vol. 43, no. 10 , p. 576-577. DOI: 10.1049/el:20070274

[4] HE, J., WANG, B. Z., HE, Q. Q., et al. Wideband X-band microstrip Butler matrix. Progress in Electromagnetics Research, 2007, vol. 74, p. 131-140. DOI: 10.2528/PIER07042302

[5] GRUSZCZYNSKI, S., WINCZA, K. Broadband $4 \times 4$ Butler matrices as a connection of symmetrical multisection coupled-line $3-\mathrm{dB}$ directional couplers and phase correction networks. IEEE Transactions on Microwave Theory and Techniques, 2009, vol. 57, no. 1, p. 1-9. DOI: 10.1109/TMTT.2008.2009081

[6] TRINH-VAN, S., LEE, J. M., YANG, Y., et al. A sidelobereduced, four-beam array antenna fed by a modified $4 \times 4$ Butler matrix for 5G applications. IEEE Transactions on Antennas and Propagation, 2019, vol. 67, no. 7, p. 4528-4536. DOI: 10.1109/tap.2019.2905783

[7] DING, K., KISHK, A. Wideband hybrid coupler with electrically switchable phase-difference performance. IEEE Microwave and Wireless Components Letters, 2017, vol. 27, no. 11, p. 992-994. DOI: $10.1109 / 1 \mathrm{mwc} .2017 .2750028$

[8] BARIK, R. K., PHANI KUMAR, K. V., KARTHIKEYAN, S. S. A compact wideband harmonic suppressed $10 \mathrm{~dB}$ branch line coupler using cascaded symmetric PI sections. Microwave and Optical Technology Letters, 2016, vol. 58, no. 7, p. 1610-1613. DOI: $10.1002 /$ mop. 29870

[9] KIM, T., LEE, J., CHOI, J. Analysis and design of miniaturized multisection crossover with open stubs. Microwave and Optical Technology Letters, 2015, vol. 57, no. 11, p. 2673-2677. DOI: 10.1002/mop. 29417 
[10] ARIBI, T., NASER-MOGHADASI, M., SADEGHZADEH, R. A. Circularly polarized beam-steering antenna array with enhanced characteristics using UCEBG structure. International Journal of Microwave and Wireless Technologies, 2015, vol. 8, no. 6, p. 955-962. DOI: 10.1017/s1759078715000318

[11] DE MAAGT, P., GONZALO, R., VARDAXOGLOU, Y. C., et al. Electromagnetic bandgap antennas and components for microwave and (sub)millimeter wave applications. IEEE Transactions on Antennas and Propagation, 2003, vol. 51, no. 10, p. 2667-2677. DOI: 10.1109/TAP.2003.817566

[12] CHANG, C., QIAN, Y., ITOH, T. Analysis and applications of uniplanar compact photonic bandgap structures. Progress in Electromagnetics Research, 2003, vol. 41, p. 211-235. DOI: 10.2528/PIER02010890

[13] REN, H., SHAO, J., ZHOU, R., et al. Compact phased array antenna system based on dual-band operations. Microwave and Optical Technology Letters, 2014, vol. 56, no. 6, p. 1391-1396. DOI: $10.1002 /$ mop. 28343

[14] KHAJEH MOHAMMAD LOU, R., NASER-MOGHADASI, M., SADEGHZADEH, R. A. Broadband planar aperture-coupled antenna array for WLAN and ITS beam-steering applications. Radio Science, 2018, vol. 53, no. 2, p. 200-209. DOI: 10.1002/2016RS006155

\footnotetext{
About the Authors ...

Mostafa MALEKI (corresponding author) was born in Urmia, Iran, in 1985. He received his B.Sc. degree in Electrical-Telecommunications Engineering, in 2008, and M.Sc. degree in Electrical Engineering, in 2013, from Islamic Azad University, and Ph.D. degree in ElectricalTelecommunications Engineering from Urmia University, Urmia, Iran, in 2020. His current research interests include
}

RF amplifiers, microwave components and high-frequency networks.

Javad NOURINIA was born in Urmia, Iran, in 1969. He received his B.Sc. degree in Electrical-Electronic Engineering from Shiraz University, M.Sc. degree in ElectricalTelecommunication Engineering from the Iran University of Science and Technology, and Ph.D. degree in ElectricalTelecommunication from the University of Science and Technology, Tehran, Iran in 2000. From 2000 he was an assistant professor and now he is a professor in the Department of Electrical Engineering of Urmia University, Urmia, Iran. His primary research interests are in antenna design, numerical methods in electromagnetic, microwave circuits.

Changiz GHOBADI was born in Iran, in 1960. He received his B.Sc. degree in Electrical Engineering, M.Sc. degrees in Electrical-Telecommunication Engineering from Isfahan University of Technology, Isfahan, Iran and Ph.D. degree in Electrical-Telecommunication from the University of Bath, Bath, UK in 1998. From 1998 he was an assistant professor and now he is a professor in the Department of Electrical Engineering of Urmia University, Urmia, Iran. His primary research interests are in antenna design, radar and adaptive filters.

Rahim NADERALI was born in Urmia, Iran, in 1963. He received his B.Sc. in Physics from Urmia University, in 1990, M.Sc. degree from Tabriz University, in 1992, and Ph.D. degree from Urmia University, in 2010. He is now an associate professor in the Urmia University, Urmia, Iran. 